\title{
The Fatty Degeneration of the Lumbar Erector Spinae Muscles Affects Dynamic Spinal Compensation Ability During Gait in Adult Spinal Deformity
}

Kousei Miura ( $\boldsymbol{\nabla}$ kmiura@tsukuba-seikei.jp )

University of Tsukuba

Hideki Kadone

University of Tsukuba Hospital

Tomoyuki Asada

University of Tsukuba

Masao Koda

University of Tsukuba

Toru Funayama

University of Tsukuba

Hiroshi Takahashi

University of Tsukuba

Hiroshi Noguchi

University of Tsukuba

Kentaro Mataki

University of Tsukuba

Yosuke Shibao

University of Tsukuba

Kosuke Sato

University of Tsukuba

Fumihiko Eto

University of Tsukuba

Mamoru Kono

University of Tsukuba

Kenji Suzuki

University of Tsukuba

Masashi Yamazaki

University of Tsukuba 


\section{Research Article}

Keywords: magnetic resonance imaging (MRI), adult spinal deformity (ASD), spinae muscles, dynamic spinal compensation, adult spinal deformity, gait

Posted Date: April 22nd, 2021

DOl: https://doi.org/10.21203/rs.3.rs-441858/v1

License: (c) (i) This work is licensed under a Creative Commons Attribution 4.0 International License.

Read Full License 


\section{Abstract}

This study aimed to investigate whether fat infiltration in lumbar back muscles assessed by magnetic resonance imaging (MRI) could be related to dynamic sagittal spino-pelvic balance during gait in adult spinal deformity (ASD). This is a retrospective analysis of 28 patients with ASD. The fat infiltration rate of lumbar erector spinae muscles and multifidus muscles was measured by T2 weighted axial MRI at L1-2 and L4-5. Dynamic sagittal spinal and pelvic angles during gait were evaluated using 3D motion analysis. The correlation between fat infiltration rate of those muscles with variations in dynamic kinematic variables while walking and static radiological parameters was analyzed. Spinal kyphosis and pelvic anteversion significantly increased during gait. Fat infiltration rate of erector spinae muscles at L1-2 was positively correlated with thoracic kyphosis $(r=0.392, p=0.039)$ and pelvic tilt $(r=0.415, p=0.028)$. Increase of spinal kyphosis during walking was positively correlated with fat infiltration rate of erector spinae muscles both at L1-2 $(r=0.394, p=0.038)$ and $L 4-5(r=0.428, p=0.023)$. Qualitative evaluation of lumbar erector spinae muscles assessed by fat infiltration rate has the potential to reflect dynamic spino-pelvic balance during gait.

\section{Introduction}

Full-spine lateral standing radiographs are used widely to evaluate adult spinal deformities (ASD). To date, sagittal spino-pelvic parameters such as sagittal vertical axis (SVA), lumbar lordosis (LL), pelvic tilt (PT) and pelvic incidence (PI) are calculated to diagnose and consider surgical strategies for patients with ASD ${ }^{1-3}$. On the other hand, there has been increasing awareness of the dynamic changes of sagittal spino-pelvic alignment. That is because a full-spine lateral standing radiograph is the only static evaluation in a transient standing position. Moreover, several sagittal spino-pelvic parameters are affected by patient positioning ${ }^{4}$. In general, low back pain and gait difficulty in ASD patients tend to increase during continuous standing or walking. Questionnaires about health-related quality of life (HRQOL) such as the Oswestry Disability Index (ODI) and short form 36-item health survey (SF-36) that are commonly used to evaluate clinical outcomes for ASD include many items related to dynamic motion.

To resolve issues related to static evaluations, three-dimensional (3D) gait analysis using motion capture systems for ASD patients have been used recently to evaluate dynamic spino-pelvic balance. A few clinical studies using gait motion analysis have reported that dynamic sagittal spino-pelvic balance deteriorated during walking in patients who have thoracolumbar spinal deformity ${ }^{5,6}$, indicating that dynamic evaluation of spinal sagittal balance may be an important addition to conventional full-spine lateral standing radiographs. However, it is difficult to perform 3D gait analysis as it requires special, and not always available, instrumentation.

Many approaches using various types of image examination have been proposed to evaluate the relationship between thoracolumbar kyphotic deformity and lumbar back muscles. Weakness of lumbar extensor muscles and marked atrophy of these muscles with fat infiltration can be demonstrated by isokinetic measurement and computed tomography (CT) scanning in patients with lumbar degenerative 
kyphosis ${ }^{7}$. A study using enhanced echo intensity on an ultrasound image of skeletal muscles found that increased intramuscular adipose tissue negatively correlated with muscle strength ${ }^{8}$. Patients with lumbar degenerative kyphosis have less muscularity of the lumbar extensor muscles and a higher proportion of fat deposits in these muscles compared to patients without spinal deformity, assessed using magnetic resonance imaging (MRI) ${ }^{9}$. Fat infiltration in back muscles has been considered a qualitative evaluation of the muscles. Although the relationship between static spinal alignment and back muscles has been reported recently in ASD patients, few studies have reported the relationship between dynamic sagittal spino-pelvic balance during gait and lumbar back muscles.

We hypothesized that the severity of fat infiltration in lumbar back muscles might reflect sagittal spinopelvic imbalance during gait for patients with ASD. If so, the qualitative evaluation of lumbar back muscles might be an alternative option for assessing the dynamic spino-pelvic balance by threedimensional gait motion analysis. The aim of this study was to investigate whether fat infiltration in lumbar back muscles assessed by MRI is related to dynamic sagittal spino-pelvic balance evaluated by three-dimensional gait motion analysis in ASD patients.

\section{Methods}

\section{Subjects}

A single institutional retrospective analysis was performed using MRI and 3D gait analysis data in ASD patients. ASD was diagnosed based on radiographic inclusion criteria measured on a full-spine lateral standing radiograph: $\mathrm{PI}-\mathrm{LL}>10^{\circ}$; SVA $>4 \mathrm{~cm}$; and $\mathrm{PT}>20^{\circ}$ as parameters defining global sagittal malalignment according to the SRS-Schwab ASD Classification ${ }^{1}$. The inclusion criteria were ASD patients who fulfilled at least one of the classification criteria and underwent 3D gait analysis and lumbar MRI between 2015 and 2019. Patients who had problems walking caused by conditions such as severe lower limb arthritis, paresis or cardiopulmonary dysfunction were excluded from the present series. Approval from the ethics committee of our local ethics committee (Tsukuba Clinical Research and Development Organization)(approval number: H26-144) was obtained for this study design. The present study was performed in accordance with the contemporary amendments of the Declaration of Helsinki and within an appropriate ethical framework. Informed consent for both study participation and publication of identifying information/images in an online open-access publication was obtained from all patients.

\section{Radiological measurements}

Full-spine lateral standing radiographs were taken to evaluate static spino-pelvic alignment. Each patient was requested to place their hands on their clavicle and stand comfortably. The static spino-pelvic parameters thoracic kyphosis (TK), SVA, LL, PT, and PI-LL were measured (Fig. 1).

\section{Measurement of fat infiltration in lumbar back muscles on MRI}


Muscle measurement data were collected with a 1.5T MRI scanner (Achieva, Philips Healthcare, Eindvohen, The Netherlands). T2 weighted axial MRI images were obtained at the L1-2 and L4-5 levels parallel to the intervertebral disc to measure fat infiltration of the lumbar back muscles. Image $J$ (National Institutes of Health, Bethesda, MD, USA) software, which can distinguish fat tissue from muscle tissue by the differences of pixel brightness and calculates the number of pixels within assigned areas on the MRI image, was used for measurement. The regions of interest (ROI) around the bilateral erector spinae muscles and multifidus muscles at those levels were manually traced. A pixel grey-scale value $>120$ on Image J was considered fat tissue in the lumbar paraspinal muscles ${ }^{10}$. The fat infiltration rate was defined as the proportion of the averaged right and left side areas of intramuscular fat tissue to the ROI of those muscles ${ }^{11,12}$ (Fig. 2).

\section{Three-dimensional gait analysis}

A laboratory with a $25 \mathrm{~m}$-long oval-shaped flat walkway was available in our hospital for gait analysis. Gait motion was analyzed using a Vicon MX system (Vicon, Oxford, UK). The VICON 16-camera system tracked movement of reflective markers attached to the spinous processes and pelvis of the patients. The patients were instructed to walk at a comfortable pace for as long as possible on the oval-shaped walkway until they stopped walking because of fatigue. We evaluated dynamic spinal and pelvic sagittal angles from this motion capture system. Dynamic spino-pelvic parameters were defined as follows: the sagittal spinal angle (SpA, the sagittal angle between the line connecting the reflective markers on the $\mathrm{C} 7$ and $\mathrm{S} 1$ spinous process and the line connecting reflective markers on the anterior superior iliac spine (ASIS) and the posterior superior iliac spine (PSIS)); and the sagittal pelvic angle (PA, the sagittal angle between the horizontal axis and the line connecting the reflective markers on the ASIS and PSIS) (Fig. 3).

\section{Statistical analysis}

Wilcoxon signed-ranks tests were used to compare dynamic spino-pelvic kinematic variables (SpA and PA) between the first lap and the final lap on the walkway. Spearman correlation coefficients were used to analyze the relationship between the fat infiltration rate of the two back muscles with the variations in dynamic spino-pelvic kinematic variables while walking $(\triangle \mathrm{SpA}$ and $\triangle \mathrm{PA})$, and with the static radiological spino-pelvic parameters (TK, LL, PT and PI-LL). All statistical analyses were performed using JMP (version 14.0.0; SAS Institute Inc, Cary, NC, USA). P values $<0.05$ were considered significant.

\section{Results}

\section{Patient demographics}

A total of 28 patients ( 8 male and 20 female) with ASD were enrolled in the present study. The mean age of the patients was $69 \pm 7.6$ years old (range 55-84 years old) and mean the BMI was $23.3 \pm 3.0$ (range 17.1-29.7).

\section{Radiological evaluation}


The mean values for static radiological spino-pelvic parameters measured from full-spine lateral standing radiographs were: SVA, $108 \pm 52 \mathrm{~mm}$ (range $-11-238 \mathrm{~mm}) ; \mathrm{TK}, 23^{\circ} \pm 16^{\circ}\left(\right.$ range $\left.-4.4^{\circ}-57^{\circ}\right) ; \mathrm{LL}, 12^{\circ} \pm$ $18^{\circ}\left(\right.$ range $\left.-21^{\circ}-49^{\circ}\right) ; \mathrm{PT}, 33^{\circ} \pm 13^{\circ}\left(\right.$ range $\left.7^{\circ}-63^{\circ}\right) ; \mathrm{PI}, 48^{\circ} \pm 12^{\circ}\left(\right.$ range $\left.18^{\circ}-71^{\circ}\right) ; \mathrm{PI}-\mathrm{LL}, 36^{\circ} \pm 17^{\circ}$ (range $1^{\circ}-75^{\circ}$ ).

\section{Evaluation of fat infiltration rate in lumbar back muscles}

The mean fat infiltration rate of erector spinae muscles at the L1-2 and L4-5 levels were $11.4 \pm 11.8 \%$ and $12.9 \pm 9.7 \%$, respectively. The mean fat infiltration rate of multifidus muscles at these levels were $18.5 \pm$ $15.2 \%$ and $24.1 \pm 16.8 \%$, respectively. The fat infiltration rate of multifidus muscles was significantly greater than that of the erector spinae muscles at both levels $(p<0.01)$.

\section{Dynamic spino-pelvic balance evaluation}

The mean SpA was $17.8^{\circ} \pm 11.8^{\circ}$ after the first lap and $19.5^{\circ} \pm 12.1^{\circ}$ after the final lap $(p<0.01)$. The mean PA was $2.93^{\circ} \pm 7.94^{\circ}$ after the first lap, and $4.39^{\circ} \pm 8.38^{\circ}$ after the final lap $(p<0.01)$. Spinal kyphosis and pelvic anteversion significantly increased after patients walked to fatigue.

\section{Correlation analysis}

The fat infiltration rate of erector spinae muscles at L1-2 was significantly correlated with TK $(r=0.392, p$ $=0.039)$ and PT $(r=0.415, p=0.028)$ (Table 1$)$. In contrast, there was no correlation between the fat infiltration rate of multifidus muscles and static spino-pelvic parameters. The increase of spinal kyphosis during walking was positively correlated with the fat infiltration rate of the erector spinae muscles both at L1-2 $(r=0.394, p=0.038)$ and at L4-5 $(r=0.428, p=0.023)$ (Table 2).

\section{Discussion}

In general, decrease of thoracic kyphosis and increase of pelvic retroversion work as compensatory mechanisms for decreased lumbar lordosis in ASD patients ${ }^{13}$. Static sagittal spino-pelvic parameters measured by full-spine lateral standing radiographs are influenced by transient compensations for spinal deformity and may underestimate spinal balance ${ }^{5}$. As previously reported, 3D gait analysis demonstrated that spinal kyphosis and pelvic anteversion significantly increased while walking and detected a failure of compensation for ASD ${ }^{6}$. Although 3D gait analysis could be optimal for quantitatively evaluating spinal balance, it has a high initial cost, making its broad use difficult. Thus, another method of static evaluation that can predict the dynamic change of spinal balance is needed. We focused on analyzing fatty degeneration of the lumbar back muscles using MRI, which is both convenient and static. As a result, the current analysis revealed significant positive correlation between increased spinal kyphosis during gait and fat infiltration in the lumbar erector spinae muscles (Fig. 4).

There has been increasing recognition of the benefits of evaluating the back muscles in patients with spinal disorders such as spinal deformity. Recent studies have mainly reported the relationship between fatty degeneration of the back muscles and static radiological spino-pelvic parameters. Fat infiltration in 
the lumbar back muscles has been correlated with several static spino-pelvic parameters including SVA ${ }^{14}, \mathrm{PT}{ }^{15}$ and $\mathrm{LL}^{16}$. Furthermore, it also has been reported that fatty degeneration of lumbar back muscles is correlated with postoperative outcomes for ASD. The increase of fat infiltration in back muscles assessed by preoperative MRI may predict proximal junctional kyphosis ${ }^{17}$ and nonunion of the lumbosacral junction ${ }^{18}$. In addition, a very limited number of studies have reported on the correlation between fatty degeneration of the back muscles and the dynamic spinal balance while walking. Lee et al. reported that dynamic spinal balance parameters assessed using 3D gait analysis showed greater correlation with fat infiltration of the lumbar paraspinal extensor muscles than with static radiological parameters ${ }^{19}$. It seems that the correlation between fatty degeneration of the back muscles and the dynamic spinal balance while walking might be important because symptoms such as back pain and gait difficulty in ASD patients increase while walking.

In the present study, the fat infiltration rate of the lumbar erector spinae muscles at L1-2 was significantly and positively correlated with thoracic kyphosis and pelvic tilt. These observations led us to speculate that increased fatty degeneration in lumbar erector spinae muscles could weaken spinal compensation for lumbar kyphosis, preventing decreased thoracic kyphosis. Pelvic compensation was not prevented, resulting in the increase of pelvic tilt. On the other hand, the increase of spinal kyphosis during walking was positively correlated with the fat infiltration rate of the lumbar erector spinae muscles at both L1-2 and L4-5. The increased fatty degeneration in lumbar erector spinae muscles could cause severe failure of spinal compensation during walking in ASD. Thus, evaluation of the fat infiltration of lumbar erector spinae muscles has the potential to reflect the dynamic change in spinal sagittal balance in ASD patients. In addition to conventional static radiological examination, it might be useful to detect individual differences in dynamic spinal compensation by analyzing the fatty condition of the lumbar erector spinae muscles to better understand the individual pathophysiology of each ASD patient. This may result in better clinical outcomes following corrective surgery for ASD.

There are several limitations to this study. First, the study is limited by the small sample size. Additionally, we evaluated fat infiltration only of the lumbar back muscles. It has been reported that fatty degeneration of hip and knee joint muscles is correlated with static radiological spino-pelvic parameters ${ }^{20}$. Hence, fat infiltration of hip and knee joint muscles may be correlated with dynamic spino-pelvic balance. Further, back muscle strength and sarcopenia, which may affect the evaluation of back muscles as well, were not assessed in this study. A prospective study with a larger sample using these variables should be completed in the future to elucidate the role of those muscles for dynamic spino-pelvic balance.

\section{Conclusions}

In this study, the fat infiltration rate of lumbar back muscles was compared with static spino-pelvic alignment parameters and dynamic spino-pelvic balance. The fat infiltration rate of lumbar erector spinae muscles at L1-2 was positively correlated with thoracic kyphosis and pelvic tilt, indicating that increased fatty degeneration in lumbar erector spinae muscles could weaken spinal compensation and maintain 
pelvic compensation for lumbar kyphosis. That caused the increase of pelvic tilt and the prevention of decreased thoracic kyphosis in ASD patients with more severe fatty degeneration of lumbar erector spinae muscles. Moreover, the fat infiltration rate of the lumbar erector spinae muscles both at L1-2 and L4-5 was positively correlated with the increase of spinal kyphosis during walking assessed using 3D gait analysis, indicating that more severe failure of spinal compensation during walking could occur in ASD patients with greater fatty degeneration of the lumbar erector spinae muscles. These findings suggest that the qualitative evaluation of lumbar erector spinae muscles assessed by fat infiltration rate has the potential to reflect the ability for spinal compensation in individual ASD patients. In addition to conventional static radiological examination, it might be useful to detect individual differences in spinal compensation by analyzing the fatty condition of the lumbar erector spinae muscles.

\section{Declarations}

\section{Funding}

This work was supported by JSPS KAKENHI Grant Number 19K18520

\section{Ethics declarations}

The authors have no conflicts of interest to declare that are relevant to the content of this article.

\section{Author contributions}

K.M. conceived this study. Mas.K., Ke.S., M.Y. contributed to the study design. K.M., H.K., T.A. coordinated the data collection and conducted analysis and data interpretation. K.M. drafted the initial manuscript.

T.F., H.T., H.N., K.M., Y.S, Ko.S., F.E, Mam.K, gave critical comments on the draft of the manuscript. All authors read and approved the final version of the manuscript.

\section{References}

1. Schwab, F. et al. Scoliosis Research Society-Schwab adult spinal deformity classification: a validation study. Spine (Phila Pa 1976) 37, 1077-1082 (2012).

2. Yamato, Y. et al. Calculation of the Target Lumbar Lordosis Angle for Restoring an Optimal Pelvic Tilt in Elderly Patients With Adult Spinal Deformity. Spine (Phila Pa 1976) 41, E211-217 (2016).

3. Inami, S. et al. Optimum pelvic incidence minus lumbar lordosis value can be determined by individual pelvic incidence. Eur Spine J 25, 3638-3643 (2016).

4. Obeid, I. et al. Global tilt: a single parameter incorporating spinal and pelvic sagittal parameters and least affected by patient positioning. Eur Spine J 25, 3644-3649 (2016).

5. Shiba, Y. et al. Dynamic global sagittal alignment evaluated by three-dimensional gait analysis in patients with degenerative lumbar kyphoscoliosis. Eur Spine J 25, 2572-2579 (2016). 
6. Miura, K. et al. Thoracic kyphosis and pelvic anteversion in patients with adult spinal deformity increase while walking: analyses of dynamic alignment change using a three-dimensional gait motion analysis system. Eur Spine J 29, 840-848 (2020).

7. Takemitsu, Y., Harada, Y., Iwahara, T., Miyamoto, M. \& Miyatake, Y. Lumbar degenerative kyphosis. Clinical, radiological and epidemiological studies. Spine (Phila Pa 1976) 13, 1317-1326 (1988).

8. Fukumoto, Y. et al. Skeletal muscle quality assessed from echo intensity is associated with muscle strength of middle-aged and elderly persons. Eur J Appl Physio/ 112, 1519-1525 (2012).

9. Kang, C. H., Shin, M. J., Kim, S. M., Lee, S. H. \& Lee, C. S. MRI of paraspinal muscles in lumbar degenerative kyphosis patients and control patients with chronic low back pain. Clin Radio/ 62, 479486 (2007).

10. Ranson, C. A., Burnett, A. F., Kerslake, R., Batt, M. E. \& O'Sullivan, P. B. An investigation into the use of MR imaging to determine the functional cross sectional area of lumbar paraspinal muscles. Eur Spine J 15, 764-773 (2006).

11. Kim, H. et al. Asymmetry of the cross-sectional area of paravertebral and psoas muscle in patients with degenerative scoliosis. Eur Spine J 22, 1332-1338 (2013).

12. Takayama, K. et al. New Predictive Index for Lumbar Paraspinal Muscle Degeneration Associated With Aging. Spine (Phila Pa 1976) 41, E84-90 (2016).

13. Lamartina, C. \& Berjano, P. Classification of sagittal imbalance based on spinal alignment and compensatory mechanisms. Eur Spine J 23, 1177-1189 (2014).

14. Park, J. S., Park, Y. S., Kim, J., Hur, J. \& Choe, D. H. Sarcopenia and fatty degeneration of paraspinal muscle associated with increased sagittal vertical axis in the elderly: a cross-sectional study in 71 female patients. Eur Spine J 29, 1353-1361 (2020).

15. Kim, D. H., Lee, S. Y., Park, S. J. \& Lee, Y. S. Relationships between Spinal Sarcopenia and Spinal Sagittal Balance in Older Women. Ann Geriatr Med Res 23, 141-148 (2019).

16. Tamai, K. et al. The evaluation of lumbar paraspinal muscle quantity and quality using the Goutallier classification and lumbar indentation value. Eur Spine J 27, 1005-1012 (2018).

17. Hyun, S. J., Kim, Y. J. \& Rhim, S. C. Patients with proximal junctional kyphosis after stopping at thoracolumbar junction have lower muscularity, fatty degeneration at the thoracolumbar area. Spine $J$ 16, 1095-1101 (2016).

18. Lee, C. S., Chung, S. S., Choi, S. W., Yu, J. W. \& Sohn, M. S. Critical length of fusion requiring additional fixation to prevent nonunion of the lumbosacral junction. Spine (Phila Pa 1976) 35, E206211 (2010).

19. Lee, J. H. \& Lee, S. H. Does lumbar paraspinal muscles improve after corrective fusion surgery in degenerative flat black? Indian J Orthop 51, 147-154 (2017).

20. Bao, H. et al. Spino-femoral muscles affect sagittal alignment and compensatory recruitment: a new look into soft tissues in adult spinal deformity. Eur Spine J 29, 2998-3005 (2020). 


\section{Tables}

Table 1.

Relationship between the fat infiltration rate in lumbar back muscles and static radiological spino-pelvic parameters

\begin{tabular}{|c|c|c|c|c|c|c|}
\hline & & SVA & TK & LL & PT & PI-LL \\
\hline \multicolumn{7}{|c|}{ Erector spinae muscles } \\
\hline \multirow[t]{2}{*}{ L1-2 } & $r$ & 0.026 & 0.393 & 0.033 & 0.415 & 0.135 \\
\hline & $\mathrm{p}$-value & 0.895 & $0.039 *$ & 0.867 & $0.028 *$ & 0.493 \\
\hline \multirow[t]{2}{*}{ L4-5 } & $r$ & 0.051 & 0.292 & -0.019 & 0.156 & 0.031 \\
\hline & p-value & 0.798 & 0.131 & 0.924 & 0.429 & 0.877 \\
\hline \multicolumn{7}{|c|}{ Multifidus muscles } \\
\hline \multirow[t]{2}{*}{ L1-2 } & $r$ & -0.001 & 0.315 & 0.020 & 0.209 & 0.027 \\
\hline & p-value & 0.995 & 0.103 & 0.920 & 0.285 & 0.892 \\
\hline \multirow[t]{2}{*}{ L4-5 } & $r$ & -0.014 & 0.367 & 0.196 & 0.339 & 0.070 \\
\hline & $\mathrm{p}$-value & 0.944 & 0.050 & 0.318 & 0.077 & 0.724 \\
\hline \multicolumn{7}{|c|}{$\begin{array}{l}\text { Abbreviations: SVA, sagittal vertical axis; TK, thoracic kyphosis; LL, lumbar lordosis; PT, pelvic tilt; PI- } \\
\text { LL, pelvic incidence minus lumbar lordosis; } r \text {, correlation coefficient. }\end{array}$} \\
\hline
\end{tabular}


Table 2.

Relationship between the fat infiltration rate in lumbar back muscles and the variations in dynamic spino-pelvic kinematic variables

\begin{tabular}{|c|c|c|c|}
\hline & & $\Delta \mathrm{SpA}$ & $\triangle \mathrm{PA}$ \\
\hline \multicolumn{4}{|c|}{ Erector spinae muscles } \\
\hline \multirow[t]{2}{*}{ L1-2 } & $r$ & 0.394 & 0.330 \\
\hline & $p$-value & $0.038 *$ & 0.086 \\
\hline \multirow[t]{2}{*}{ L4-5 } & r & 0.428 & 0.239 \\
\hline & $\mathrm{p}$-value & $0.023 *$ & 0.222 \\
\hline \multicolumn{4}{|c|}{ Multifidus muscles } \\
\hline \multirow[t]{2}{*}{ L1-2 } & $r$ & 0.220 & 0.292 \\
\hline & $\mathrm{p}$-value & 0.260 & 0.132 \\
\hline \multirow[t]{2}{*}{ L4-5 } & $r$ & 0.314 & 0.100 \\
\hline & $\mathrm{p}$-value & 0.104 & 0.613 \\
\hline \multicolumn{4}{|c|}{$\begin{array}{l}\text { Abbreviations: SpA, sagittal spinal angle; } \mathrm{PA} \text {, sagittal pelvic angle; } r \text {, correlation coefficient } \\
\star_{\mathrm{D}<0}<5\end{array}$} \\
\hline
\end{tabular}

Figures 


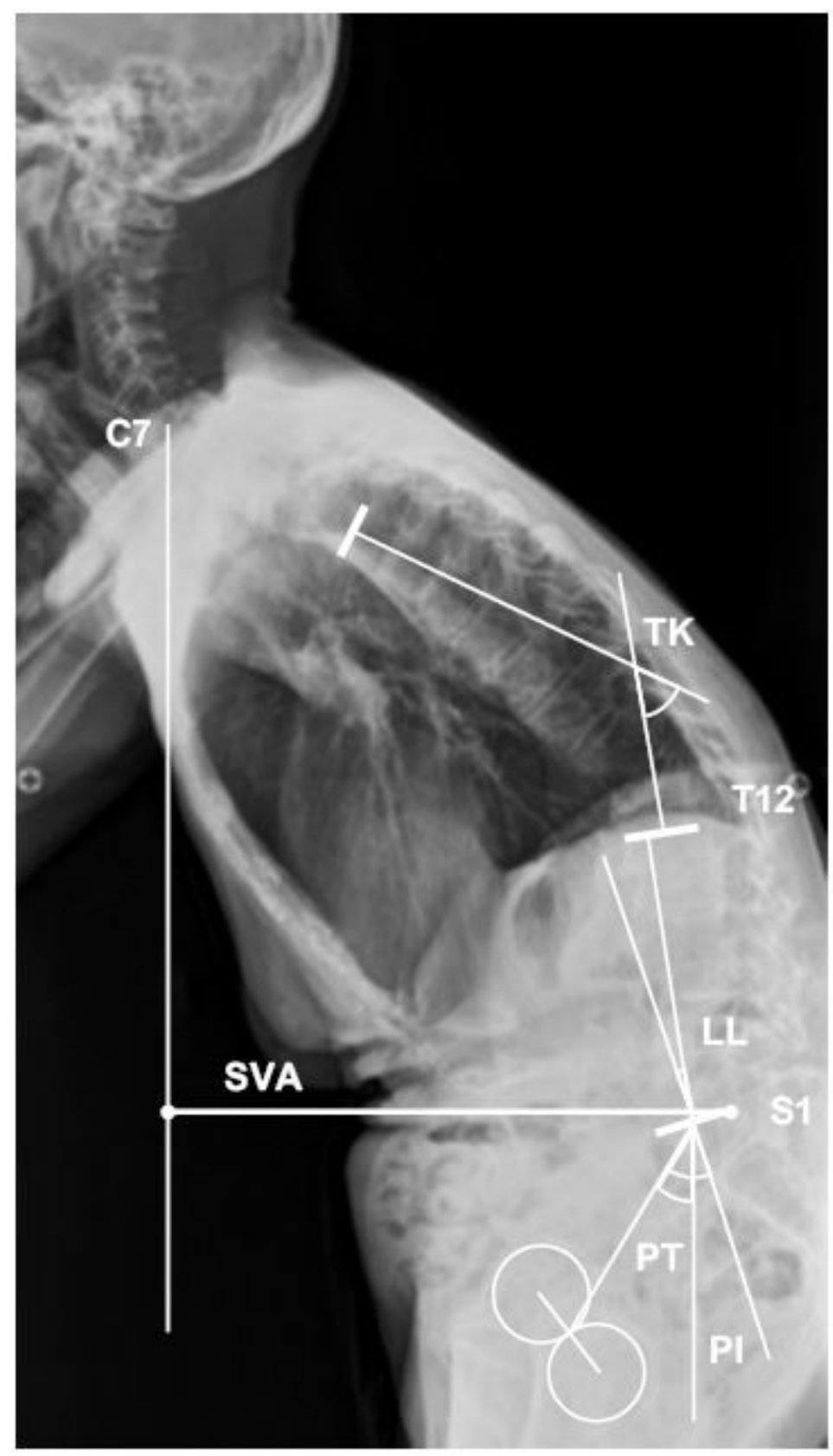

\section{Figure 1}

Static spino-pelvic parameters measured by full-spine lateral standing radiographs. SVA, the sagittal vertical axis; TK, thoracic kyphosis (T5-12); LL, lumbar lordosis (T12-S1); PT, pelvic tilt; PI, pelvic incidence 

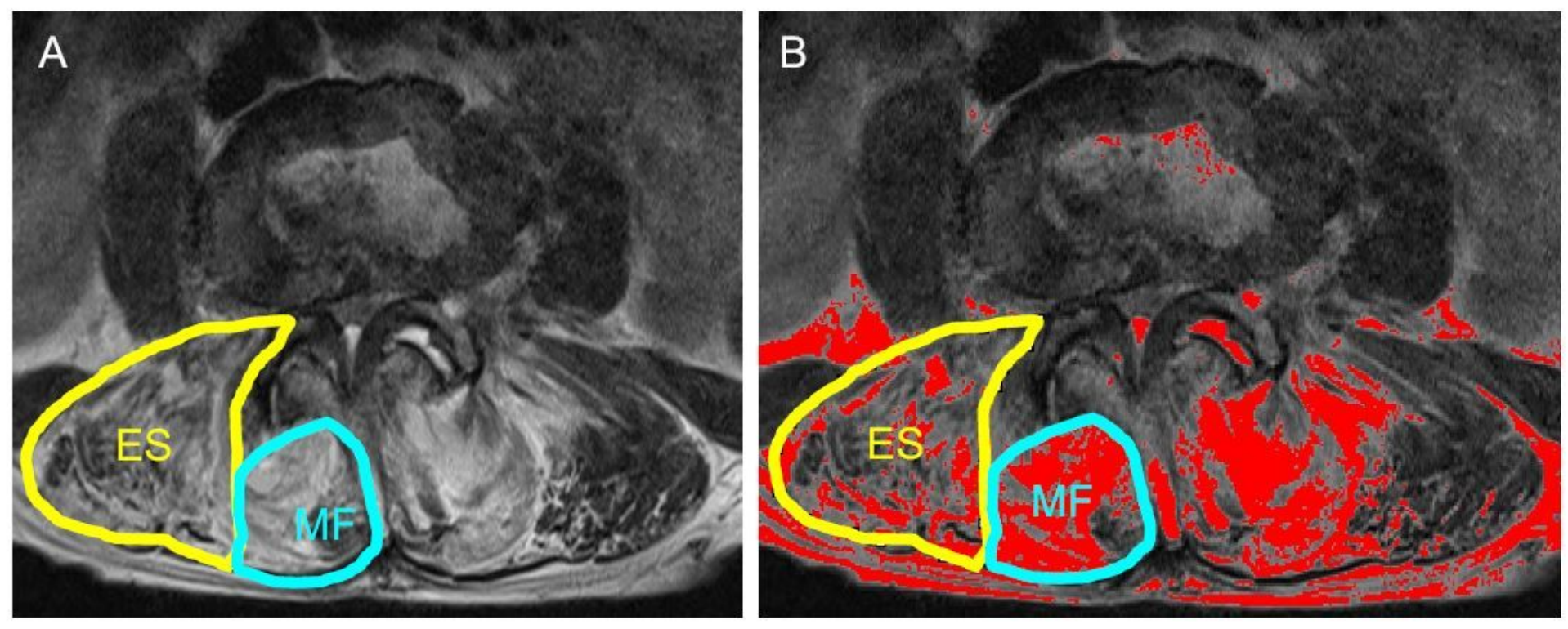

\section{Figure 2}

Calculation of the fat infiltration rate defined as the proportion of the area of the intramuscular fat tissue to the area of the regions of interest (ROI) of lumbar back muscles. A: Manually traced regions of interest (ROI) around the erector spinae muscles and multifidus muscles at the L1-2 and L4-5 disc levels on axial T2 weighted MRI images. B: The intramuscular red area showing the fat signal area in the lumbar back muscles by using Image $\mathrm{J}$ software (National Institutes of Health, Bethesda, MD, USA). ES, erector spinae muscles; MF, multifidus muscles. 

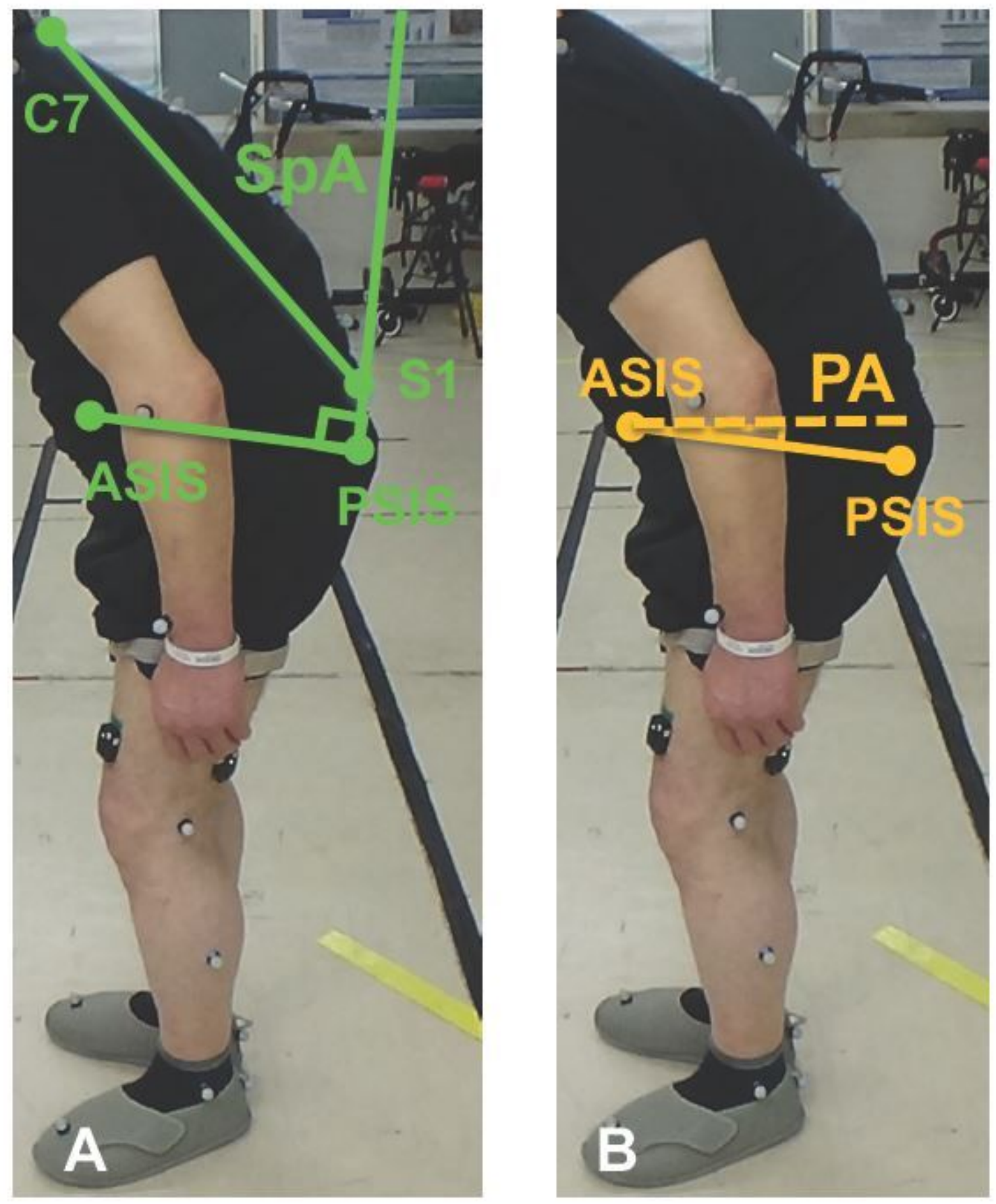

\section{Figure 3}

Measurement of dynamic spino-pelvic sagittal balance during gait from the three-dimensional motion analysis. A: The sagittal spinal angle ( $\mathrm{SpA}$ ) is the sagittal angle between the line connecting the reflective markers on the $\mathrm{C} 7$ and $\mathrm{S} 1$ spinous process and the line connecting reflective markers on the anterior superior iliac spine (ASIS) and the posterior superior iliac spine (PSIS)). B: The sagittal pelvic angle (PA) is the sagittal angle between the horizontal axis and the line connecting the reflective markers on the ASIS and PSIS) 


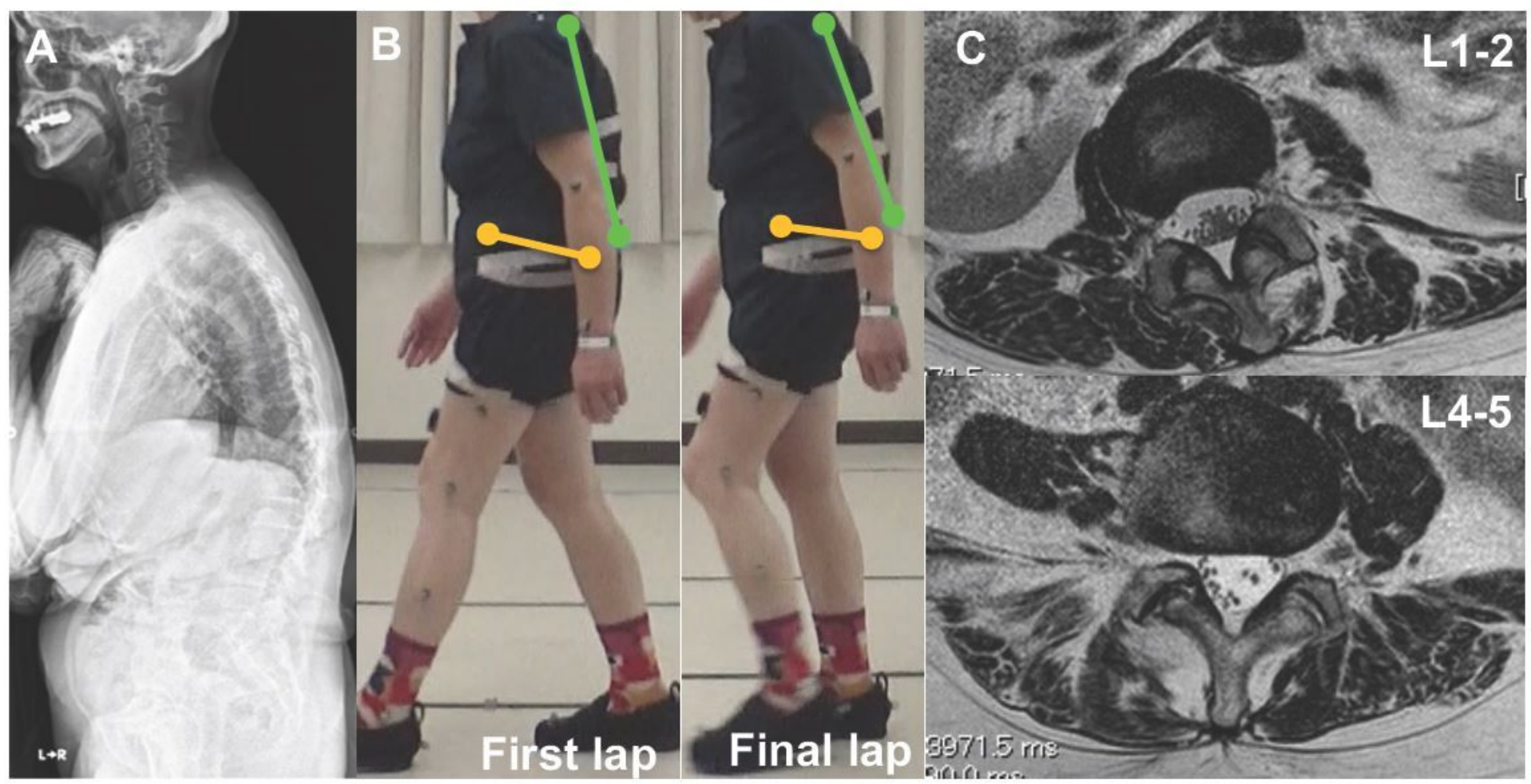

Figure 4

A representative case of a 63-year-old female patient with almost normal TK and increased PT, large fat infiltration rate of the lumbar erector spinae muscles and an increase of spinal kyphosis and pelvic anteversion. A: Full-spine lateral radiograph. SVA: $89 \mathrm{~mm}$, TK: 40 ${ }^{\circ}$ LL5.8 ${ }^{\circ}, \mathrm{PT} 35^{\circ}$, PI: $39^{\circ}$ B: Posture during the three-dimensional gait motion analysis on the first and final laps. $\triangle \mathrm{SpA}: 2.5^{\circ}, \triangle \mathrm{PA}: 3.1^{\circ} \mathrm{C}: \mathrm{T} 2$ weighted axial MRI images at L1-2 and L4-5. The fat infiltration rate of the lumbar erector spinae muscles (L1-2: $15 \%$ and L4-5: $24 \%$ ) 